\title{
Ethno-pharmacological survey of herbal remedies used in the treatment of paediatric diseases in Buhunga parish, Rukungiri District, Uganda
}

Patience Tugume* ${ }^{*}$ and Clement Nyakoojo

\begin{abstract}
Background: Plants have been used as a primary source of medicine since ancient times and about $80 \%$ of the world's population use herbal medicine to treat different ailments. Plant use knowledge differs in space and time and thus requires documentation to avoid its loss from one generation to another.

Methods: In order to accomplish the survey, semi-structured questionnaires were used. The data collected included names of plant species, parts used, ailments treated, growth habit, methods of preparation and mode of administration of the herbal remedies. Descriptive statistics were used to present the data in form of tables and a graph.

Results: Results showed that 50 plant species belonging to 26 families were utilized in the treatment of paediatric diseases of which Asteraceae and Lamiaceae were the most common. Leaves (80\%) were the most commonly used and decoctions were the main method of preparation. Twenty nine health conditions were treated out of which digestive disorders, malaria and respiratory tract infections were predominant. Herbs and shrubs were equally dominant.

Conclusion: Herbal remedies are an important source of treatment for paediatric diseases in Buhunga Parish. However, there is need for collaboration between herbal medicine users and scientific institutions to help in the discovery of new drugs based on indigenous knowledge. Scientists ought to explore suitable methods of preparation and dosage formulations in order to achieve the best benefits from herbal remedies.
\end{abstract}

Keywords: Traditional medicine, Herbal remedies, Children diseases, Buhunga, Digestive disorders

\section{Background}

Plants have been used as a source of medicines for both humans and animals since time immemorial in crude forms such as decoctions, syrups, powders, infusions and ointments [1]. The use of herbal medicine in primary health care is still practiced in both developed and developing countries [2]. According to the World Health Organization (WHO) about $80 \%$ of the populations in developing countries use herbal medicine to meet their primary healthcare requirements $[3,4]$.

Traditional medicine is considered as a total sum of all practices, measures and procedures which have from

\footnotetext{
*Correspondence: ptugume@cns.mak.ac.ug; patiebeys@gmail.com Department of Plant Sciences, Microbiology \& Biotechnology, Makerere University, P.O Box 7062, Kampala, Uganda
}

time immemorial enabled Africans guard against disease. Kamatenesi [5] emphasises the importance of indigenous knowledge on plants species in the management of diseases. According to WHO [3], traditional medicine is characterized by a diversity of uses by people in different age groups. In Africa, reliance on folk medicine is partly due to the high cost of modern medicine and inaccessibility to health facilities [6].

Most of the ethnobotanical literature on traditional medicine is concentrated on the knowledge of traditional healers and largely overlooks domestic medicine and in particular the knowledge of women [7]. Domestic knowledge on the utilisation of herbal medicines needs to be prioritized in research in order to boost the interest of local people about their role in the management of

(C) The Author(s). 2019 Open Access This article is distributed under the terms of the Creative Commons Attribution 4.0 International License (http://creativecommons.org/licenses/by/4.0/), which permits unrestricted use, distribution, and 
diseases. The use of medicinal plant species in the treatment of children diseases is part and parcel of traditional knowledge that is handed down by word of mouth or orally hence documentation is necessary.

Over seven million children under five years of age die globally each year from preventable and treatable diseases especially pneumonia, diarrhoea and malaria [8]. Such children could be saved if interventions such as the use of antibiotics for pneumonia treatment, oral rehydration therapy for diarrhoea and use of insecticide-treated nets to prevent malaria are universally affordable [8].

In Uganda, the child mortality rate due to preventable diseases is still high at 90 deaths in 1000 live births [9]. Development of diseases in children is a particularly important concern because their health could be seriously threatened $[10,11]$. In rural areas, owing to inadequate health facilities, high cost of conventional medicine, underfunding and mismanagement of the available health facilities, parents resort to the use of herbal medicine rather than modern medicine for treatment of children ailments [12,13].

Although there is increased use of herbal medicine in Uganda, it could result into complications in case of improper prescriptions and dosages [3]. Much of the knowledge on the use of medicinal plants is possessed by traditional societies whose existence is threatened due to lack of systematic documentation. In addition to loss of indigenous knowledge, many medicinal plant species are facing extinction that could lead to genetic loss hence the need to document such information. The study aimed at documenting the medicinal plant species used in the treatment of paediatric diseases and their associated indigenous knowledge so that measures might be taken to develop new low cost therapies affordable by the rural poor.

\section{Materials and methods Study area}

Buhunga Parish is located in Buhunga Sub-County, Rukungiri District in the Kigezi Sub-Region of Western Uganda. It is located between latitudes $0^{\circ} 45^{\prime} 18.7^{\prime \prime} \mathrm{S}$ and longitudes $29^{\circ} 59^{\prime} 0.9 .6^{\prime \prime} \mathrm{E}$. It is densely populated with a density of 328.8 people per square kilometre. The majority of the people are females. The parish consists of six villages namely Mutanoga, Kitookye, Rutooma, Kishaka, Nyamuyaga and Oruhita. The area is characterized by a hilly fertile landscape with occasional valleys that are dominated by livestock farms and banana plantations. There are two rainy seasons, one occurring from March to May and the other from September to November. The dominant tribes are the Banyakole and Bakiga.

\section{Methods}

An ethnobotanical survey was conducted between December 2018 and February 2019 to document the therapeutic values of plant species used to treat childhood diseases, plant parts used, methods of preparation and administration. Standard ethnobotanical procedures [14] were followed. Data collection was based on structured interviews [15] and the responses were recorded by the researcher. The interviews were conducted in the local language of the participants since most of them were not formally educated. All interviews were conducted after obtaining verbal prior informed consent from participants. The first section of the questionnaire required demographic information of participants. The second section required local names of plant species used, diseases treated, modes of preparation and administration of the herbal recipes, plant part(s) frequently used, growth habits and habitats of the plant species.

The lottery method was used to randomly select 10 households from each of the six villages. Household heads were purposively selected for interview because they are the ones concerned about the healthcare of families. Plant specimens used in herbal recipes were collected with the aid of respondents and authenticated using their local names and standard text [16].Voucher specimens were collected, processed and deposited at the Makerere University Herbarium. Correctness of scientific names was checked in the database of International Plant Names Index (IPNI) https://www.ipni.org accessed on 1st February 2019.

\section{Results}

\section{Demographic information of participants}

The participants were mainly women (60\%) above 18 years old. They had low education levels whereby $50 \%$ had not attained any formal education, $40 \%$ had attained at least primary education with the balance having attained secondary education level. A majority of participants were women because they are usually involved in caring for children. Most of the women were either single parents or widowed and thus with a heavy burden of providing for their children and had not attained any formal education compared to men who had at least attained primary and secondary levels of education. The low levels of education of participants imply limited employment opportunities and hence limited income. With the limited income, they have no option except the use of herbal medicine to provide healthcare for their children.

\section{Diversity of plant species used}

The survey revealed a total of 50 medicinal plant species from 26 families were used to treat paediatric diseases. Scientific names, local names, family, growth form, 
Table 1 Medicinal plant species, their habit, habitat, parts used, ailments treated, mode of preparation and administration

\begin{tabular}{|c|c|c|c|c|c|c|c|c|}
\hline Family & $\begin{array}{l}\text { Scientific } \\
\text { name, } \\
\text { Voucher No. }\end{array}$ & $\begin{array}{l}\text { Local name } \\
\text { (Runyankole/ } \\
\text { Rukiga) }\end{array}$ & Habit & Habitat & $\begin{array}{l}\text { Parts } \\
\text { used }\end{array}$ & $\begin{array}{l}\text { Ailment } \\
\text { treated }\end{array}$ & $\begin{array}{l}\text { Method of } \\
\text { preparation \& } \\
\text { administration }\end{array}$ & $\begin{array}{l}\text { Literature supporting } \\
\text { traditional use of the } \\
\text { plant species in } \\
\text { other regions. }\end{array}$ \\
\hline Acanthaceae & $\begin{array}{l}\text { Monechma } \\
\text { subsessile C. B. } \\
\text { Clarke PT } 040\end{array}$ & Erazi & $\mathrm{H}$ & $\begin{array}{l}\text { Along foot } \\
\text { paths }\end{array}$ & L & $\begin{array}{l}\text {-Stomachache } \\
\text {-Epilepsy }\end{array}$ & -Decoction drunk & Not found \\
\hline Aloeaceae & $\begin{array}{l}\text { Aloe vera }(L) \\
\text { Burm. f. PT046 }\end{array}$ & Rukaka & S & Grasslands & L & -Stomachache & -Decoction drunk & Not found \\
\hline Anarcadiaceae & $\begin{array}{l}\text { Mangifera } \\
\text { indica L.PT032 }\end{array}$ & Omuyembe & $\mathrm{T}$ & Farmland & $\begin{array}{l}L \\
B\end{array}$ & -Cough & $\begin{array}{l}\text {-Infusion drunk } \\
\text {-Juice from } \\
\text { macerated leaves } \\
\text { drunk } \\
\text {-Decoction drunk }\end{array}$ & $\begin{array}{l}\text {-Oral wounds, tonsillitis } \\
\text { and fever [17] }\end{array}$ \\
\hline Apiaceae & $\begin{array}{l}\text { Centella } \\
\text { asiatica (L.) } \\
\text { Urb. PT011 }\end{array}$ & & $\mathrm{H}$ & $\begin{array}{l}\text { Swamps } \\
\text { Farmland }\end{array}$ & Wp & $\begin{array}{l}\text {-Dysentery } \\
\text {-Constipation } \\
\text {-Jaundice } \\
\text {-Fever }\end{array}$ & $\begin{array}{l}\text {-Infusion drunk } \\
\text {-Juice from } \\
\text { macerated leaves } \\
\text { drunk }\end{array}$ & -Boils in Madagascar [18] \\
\hline Asparagaceae & $\begin{array}{l}\text { Draceana } \\
\text { fragrans }(\mathrm{L} .) \\
\text { Ker Gawl. } \\
\text { PT008 }\end{array}$ & Omugorora & S & $\begin{array}{l}\text { Bush } \\
\text { Farmland }\end{array}$ & $\begin{array}{l}\mathrm{S}, \mathrm{L}, \\
\mathrm{R}\end{array}$ & -Stomachache & -Decoction drunk & $\begin{array}{l}\text { Leaves used for ear } \\
\text { infections in Kibale [19] }\end{array}$ \\
\hline \multirow[t]{8}{*}{ Asteraceae } & $\begin{array}{l}\text { Aspilia } \\
\text { africana (Pers) } \\
\text { adama PT } 006\end{array}$ & Ekiterankuba & $\mathrm{H}$ & $\begin{array}{l}\text { Bush } \\
\text { Farmland }\end{array}$ & L & $\begin{array}{l}\text {-Diarrhoea } \\
\text { - } \\
\text { Stomachache } \\
\text {-Epilepsy }\end{array}$ & $\begin{array}{l}\text {-Decoction drunk } \\
\text {-Juice from } \\
\text { macerated leaves } \\
\text { drunk }\end{array}$ & $\begin{array}{l}\text {-Child delay, wounds in } \\
\text { Erute county [20] }\end{array}$ \\
\hline & $\begin{array}{l}\text { Bidens pilosa } \\
\text { L. PT031 }\end{array}$ & Enyabarashana & $\mathrm{H}$ & $\begin{array}{l}\text { Bush } \\
\text { Farmland }\end{array}$ & $\begin{array}{l}L \\
R\end{array}$ & $\begin{array}{l}\text {-Fresh } \\
\text {-wounds } \\
\text {-Malaria } \\
\text {-Worms } \\
\text {-Constipation } \\
\text {-Poisoning }\end{array}$ & $\begin{array}{l}\text {-Leaves macerated } \\
\text { and applied } \\
\text { topically } \\
\text {-Raw roots } \\
\text { chewed } \\
\text {-Decoction drunk }\end{array}$ & $\begin{array}{l}\text { Also used for wounds in } \\
\text { western Uganda [21] } \\
\text { Stomachaches and } \\
\text { jaundice in Peru [22] }\end{array}$ \\
\hline & $\begin{array}{l}\text { Erlangea } \\
\text { cordifolia S. } \\
\text { Moore PT001 }\end{array}$ & Akatoma & S & $\begin{array}{l}\text { Bush } \\
\text { Abandoned } \\
\text { lands }\end{array}$ & $L$ & $\begin{array}{l}\text {-Stomachache } \\
\text {-Worms }\end{array}$ & -Decoction drunk & $\begin{array}{l}\text { Stomach upsets in newly } \\
\text { borns [19] }\end{array}$ \\
\hline & $\begin{array}{l}\text { Erlangea } \\
\text { tomentosa S. } \\
\text { Moore PT004 }\end{array}$ & Ekyoganyanja & $\mathrm{H}$ & $\begin{array}{l}\text { Bush } \\
\text { Abandoned } \\
\text { lands }\end{array}$ & L & $\begin{array}{l}\text {-Stomachache } \\
\text {-Jaundice }\end{array}$ & -Decoction drunk & $\begin{array}{l}\text { Used for colic pains and } \\
\text { stomachache in western } \\
\text { Uganda [21]; Cough [19] }\end{array}$ \\
\hline & $\begin{array}{l}\text { Vernonia } \\
\text { amygdalina } \\
\text { Delile PT010 }\end{array}$ & Omubirizi & S & $\begin{array}{l}\text { Farmland } \\
\text { Bush }\end{array}$ & $L, R$ & $\begin{array}{l}\text {-Worms } \\
\text {-Malaria } \\
\text {-Fever } \\
\text {-Indigestion }\end{array}$ & -Decoction drunk & $\begin{array}{l}\text { Burns, colic, malaria, syphilis, } \\
\text { ulcers, wounds, skin rash [21]; } \\
\text { Malaria, yellow fever [17] }\end{array}$ \\
\hline & $\begin{array}{l}\text { Vernonia } \\
\text { auriculifera } \\
\text { Hiern. PT019 }\end{array}$ & Ekinyekanyeme & S & Bush & L & $\begin{array}{l}\text {-Fever } \\
\text {-Malaria } \\
\text {-Stomachache }\end{array}$ & $\begin{array}{l}\text {-Infusion drunk } \\
\text {-Decoction drunk }\end{array}$ & Placenta removal [19] \\
\hline & $\begin{array}{l}\text { Vernonia } \\
\text { lasiopas o. } \\
\text { Hoffm PT025 }\end{array}$ & Omujuma & S & Bush & L & -Worms & $\begin{array}{l}\text {-Decoction drunk } \\
\text {-Juice from } \\
\text { macerated leaves } \\
\text { drunk }\end{array}$ & $\begin{array}{l}\text { Used against constipation, } \\
\text { deworming, malaria, skin } \\
\text { allergy, stomachache in } \\
\text { Western Uganda [21] } \\
\text { Malaria, stomachache [23] }\end{array}$ \\
\hline & $\begin{array}{l}\text { Senecio } \\
\text { ruwenzoriensis } \\
\text { S. Moore } \\
\text { PT036 }\end{array}$ & Omuzilafu & $\mathrm{H}$ & $\begin{array}{l}\text { Grasslands } \\
\text { Farmland }\end{array}$ & L & $\begin{array}{l}\text {-Stomachache } \\
\text {-Fever }\end{array}$ & $\begin{array}{l}\text {-Decoction drunk } \\
\text {-Decoction bathed }\end{array}$ & Not found \\
\hline \multirow[t]{2}{*}{ Bignoniaceae } & $\begin{array}{l}\text { Markhamia } \\
\text { lutea K. } \\
\text { Schum PT } 018\end{array}$ & Omusambya & $\mathrm{T}$ & $\begin{array}{l}\text { Bush } \\
\text { Farmland }\end{array}$ & $\mathrm{R}$ & -Diarrhoea & -Infusion drunk & Diarrhoea, gonorrhoea [19] \\
\hline & $\begin{array}{l}\text { Spathodea } \\
\text { companulata P. } \\
\text { Beauv.PT005 }\end{array}$ & Munyara & T & Bush & $L, B$ & $\begin{array}{l}\text {-Allergy } \\
\text {-Dysentery } \\
\text {-Diarrhoea } \\
\text {-Asthma }\end{array}$ & -Decoction drunk & $\begin{array}{l}\text { Infertility, skin infections, } \\
\text { Hernia [23] }\end{array}$ \\
\hline
\end{tabular}


Table 1 Medicinal plant species, their habit, habitat, parts used, ailments treated, mode of preparation and administration (Continued)

\begin{tabular}{|c|c|c|c|c|c|c|c|c|}
\hline Family & $\begin{array}{l}\text { Scientific } \\
\text { name, } \\
\text { Voucher No. }\end{array}$ & $\begin{array}{l}\text { Local name } \\
\text { (Runyankole/ } \\
\text { Rukiga) }\end{array}$ & Habit & Habitat & $\begin{array}{l}\text { Parts } \\
\text { used }\end{array}$ & $\begin{array}{l}\text { Ailment } \\
\text { treated }\end{array}$ & $\begin{array}{l}\text { Method of } \\
\text { preparation \& } \\
\text { administration }\end{array}$ & $\begin{array}{l}\text { Literature supporting } \\
\text { traditional use of the } \\
\text { plant species in } \\
\text { other regions. }\end{array}$ \\
\hline Caricaceae & $\begin{array}{l}\text { Carica papaya } \\
\text { L. PT002 }\end{array}$ & Omupapari & $\mathrm{T}$ & Farmland & $\begin{array}{l}\text { L } \\
F R \\
R\end{array}$ & $\begin{array}{l}\text {-Worms } \\
\text {-Cough } \\
\text {-Wounds }\end{array}$ & $\begin{array}{l}\text {-Decoction drunk } \\
\text {-Latex leaked } \\
\text {-Macerated leaves }\end{array}$ & $\begin{array}{l}\text {-Roots used against cough } \\
\text { and diarrhoea in Ngai [24] }\end{array}$ \\
\hline \multirow[t]{2}{*}{ Chenopodiaceae } & $\begin{array}{l}\text { Chenopodium } \\
\text { ambrosioides L. } \\
\text { PT012 }\end{array}$ & Musasizi & $\mathrm{H}$ & Bush & L & $\begin{array}{l}\text {-Headache } \\
\text {-Stomachache }\end{array}$ & $\begin{array}{l}\text { - Macerated leaves } \\
\text { rubbed in the } \\
\text { head } \\
\text {-Decoction drunk }\end{array}$ & $\begin{array}{l}\text { For endoparasites; also as } \\
\text { laxative in Peru [22]; Skin } \\
\text { swellings in India [25] }\end{array}$ \\
\hline & $\begin{array}{l}\text { Chenopodium } \\
\text { opulifolium } \\
\text { Koch \& Ziz } \\
\text { PT020 }\end{array}$ & Omwetango & $\mathrm{H}$ & Bush & L & $\begin{array}{l}\text {-Malaria } \\
\text {-Fever } \\
\text {-Misfortune }\end{array}$ & $\begin{array}{l}\text {-Infusion drunk } \\
\text {-Decoction bathed } \\
\text {-Macerated leaves } \\
\text { mixed with water } \\
\text { and child is } \\
\text { bathed }\end{array}$ & $\begin{array}{l}\text { Oral wounds, skin rash, } \\
\text { toothache, sore throat [23] }\end{array}$ \\
\hline Curcubitaceae & $\begin{array}{l}\text { Mormodica } \\
\text { feotida K. } \\
\text { Schum PT } 021\end{array}$ & Akabombo & C & $\begin{array}{l}\text { Farmland } \\
\text { Hedges }\end{array}$ & L & $\begin{array}{l}\text {-Malaria } \\
\text {-Fever } \\
\text {-Worms } \\
\text {-Cough } \\
\text {-Stomachache }\end{array}$ & $\begin{array}{l}\text {-Decoction or } \\
\text { infusion drunk }\end{array}$ & $\begin{array}{l}\text { Yellow fever, cough and } \\
\text { malaria in lganga [17] }\end{array}$ \\
\hline \multirow[t]{2}{*}{ Euphorbiaceae } & $\begin{array}{l}\text { Eurphobia hirta } \\
\text { L. PT014 }\end{array}$ & Enkukuru & $\mathrm{H}$ & $\begin{array}{l}\text { Farmland } \\
\text { Compound }\end{array}$ & $\begin{array}{l}L \\
W P\end{array}$ & $\begin{array}{l}\text {-Worms } \\
\text {-Asthma } \\
\text {-Wounds } \\
\text {-Eye infections }\end{array}$ & $\begin{array}{l}\text {-Decoction drunk } \\
\text {-Leaves burnt and } \\
\text { ash applied to } \\
\text { wounds and boils }\end{array}$ & $\begin{array}{l}\text { Sterility, diarrhoea, false teeth } \\
\text { in Bulamogi [26] }\end{array}$ \\
\hline & $\begin{array}{l}\text { Euphorbia } \\
\text { turicalli L. } \\
\text { PT030 }\end{array}$ & Oruyenje & S & $\begin{array}{l}\text { Farmland } \\
\text { Hedges }\end{array}$ & $\begin{array}{l}\mathrm{R} \\
\mathrm{Br}\end{array}$ & $\begin{array}{l}\text { Stomachache } \\
\text {-Sore throat } \\
\text {-Snake bites }\end{array}$ & $\begin{array}{l}\text {-Decoction drunk } \\
\text {-Young branches } \\
\text { roasted and } \\
\text { chewed }\end{array}$ & Sap used to treat warts [23] \\
\hline \multirow[t]{4}{*}{ Fabaceae } & $\begin{array}{l}\text { Albizia } \\
\text { adianthifoli } \\
\text { (Schumach.) W. } \\
\text { Wight PT043 }\end{array}$ & Omushebeya & $\mathrm{T}$ & Farmland & L & -Fever & -Decoction drunk & Not found \\
\hline & $\begin{array}{l}\text { Cassia } \\
\text { occidentalis } \\
\text { Linn. PT } 016\end{array}$ & Omwitanjoka & S & $\begin{array}{l}\text { Farmland } \\
\text { Bush }\end{array}$ & $\begin{array}{l}L \\
R\end{array}$ & $\begin{array}{l}\text {-Worms } \\
\text {-Stomachache } \\
\text {-Headache }\end{array}$ & $\begin{array}{l}\text {-Infusion or } \\
\text { decoction drunk }\end{array}$ & Paediatric cough [19] \\
\hline & $\begin{array}{l}\text { Mimosa pudica } \\
\text { L. PT035 }\end{array}$ & Kabarashaha & $\mathrm{H}$ & $\begin{array}{l}\text { Farmland } \\
\text { Home } \\
\text { compounds }\end{array}$ & $L, R$ & $\begin{array}{l}\text {-Sores } \\
\text {-Abcesses }\end{array}$ & $\begin{array}{l}\text {-Macerated leaves/ } \\
\text { roots applied on } \\
\text { affected part }\end{array}$ & $\begin{array}{l}\text { Pre-mature ejaculation, } \\
\text { measles [26] }\end{array}$ \\
\hline & $\begin{array}{l}\text { Sesbania } \\
\text { sesban (L.) } \\
\text { Merr PT } 013\end{array}$ & Omunyeganyeje & $\mathrm{S}$ & Farmland & L & $\begin{array}{l}\text {-Stomachache } \\
\text {-Abscesses }\end{array}$ & $\begin{array}{l}\text {-Decoction drunk } \\
\text {-Macerated leaves } \\
\text { mixed with water } \\
\text { and child bathed }\end{array}$ & $\begin{array}{l}\text { High blood pressure, } \\
\text { Diabetes [23] }\end{array}$ \\
\hline \multirow[t]{5}{*}{ Lamiaceae } & $\begin{array}{l}\text { Iboza riparia } \\
\text { N.E. Br. PT } 017\end{array}$ & Omuravunga & $\mathrm{H}$ & Farmland & L & $\begin{array}{l}\text {-Cough } \\
\text {-Stomachache }\end{array}$ & -Decoction drunk & Not found \\
\hline & $\begin{array}{l}\text { Leonotis } \\
\text { nepetifolia (L.) } \\
\text { R. Br. PT } 028\end{array}$ & Ekicumucumu & $\mathrm{H}$ & $\begin{array}{l}\text { Bush Along } \\
\text { foot paths }\end{array}$ & $\begin{array}{l}L \\
W P\end{array}$ & $\begin{array}{l}\text {-Stomachache } \\
\text {-Dysentery } \\
\text {-Convulsions } \\
\text {-Malaria } \\
\text {-Fever }\end{array}$ & $\begin{array}{l}\text {-Decoction drunk } \\
\text {-Decoction bathed }\end{array}$ & $\begin{array}{l}\text { Colic, convulsions, } \\
\text { deworming, dysentery, } \\
\text { miscarriage, uterine pains in } \\
\text { Western Uganda [21] }\end{array}$ \\
\hline & $\begin{array}{l}\text { Ocimum } \\
\text { kilimand } \\
\text { Schium PT } 042\end{array}$ & Obushonga & S & Farmland & L & $\begin{array}{l}\text {-Headache } \\
\text {-Stomachache }\end{array}$ & $\begin{array}{l}\text {-Macerated leaves } \\
\text { rubbed on the } \\
\text { head } \\
\text {-Decoction drunk }\end{array}$ & Not found \\
\hline & $\begin{array}{l}\text { Ocimum } \\
\text { gratissum L. } \\
\text { PT049 }\end{array}$ & Omujaja & S & Bush & L & -Stomachache & $\begin{array}{l}\text {-Decoction or } \\
\text { infusion drunk }\end{array}$ & Not found \\
\hline & $\begin{array}{l}\text { Ocimum suave } \\
\text { Willd. PT045 }\end{array}$ & Esitimwa & S & $\begin{array}{l}\text { Bush } \\
\text { Farmland }\end{array}$ & L & -Stomachache & $\begin{array}{l}\text {-Juice from } \\
\text { macerated leaves }\end{array}$ & $\begin{array}{l}\text { Allergy, colic constipation, } \\
\text { deworming, flue, }\end{array}$ \\
\hline
\end{tabular}


Table 1 Medicinal plant species, their habit, habitat, parts used, ailments treated, mode of preparation and administration (Continued)

\begin{tabular}{|c|c|c|c|c|c|c|c|c|}
\hline Family & $\begin{array}{l}\text { Scientific } \\
\text { name, } \\
\text { Voucher No. }\end{array}$ & $\begin{array}{l}\text { Local name } \\
\text { (Runyankole/ } \\
\text { Rukiga) }\end{array}$ & Habit & Habitat & $\begin{array}{l}\text { Parts } \\
\text { used }\end{array}$ & $\begin{array}{l}\text { Ailment } \\
\text { treated }\end{array}$ & $\begin{array}{l}\text { Method of } \\
\text { preparation \& } \\
\text { administration }\end{array}$ & $\begin{array}{l}\text { Literature supporting } \\
\text { traditional use of the } \\
\text { plant species in } \\
\text { other regions. }\end{array}$ \\
\hline & & & & & & & drunk & $\begin{array}{l}\text { stomachache, uterine pain, } \\
\text { headache, splenomegaly [27] }\end{array}$ \\
\hline & $\begin{array}{l}\text { Plectranthus } \\
\text { barbatus } \\
\text { Andr.PT027 }\end{array}$ & Rubatura & $\mathrm{H}$ & $\begin{array}{l}\text { Farmland } \\
\text { Along foot } \\
\text { paths }\end{array}$ & L & -Stomachache & -Decoction drunk & Not found \\
\hline & $\begin{array}{l}\text { Plecteanthus } \\
\text { forskahlii Willd } \\
\text { PT023 }\end{array}$ & Ekizera & $\mathrm{H}$ & Farmland & L & $\begin{array}{l}\text {-Stomachache } \\
\text {-Worms } \\
\text {-Fever } \\
\text {-Malaria }\end{array}$ & $\begin{array}{l}\text {-Infusion or } \\
\text { decoction drunk }\end{array}$ & Not found \\
\hline Malvaceae & $\begin{array}{l}\text { Sida } \\
\text { rhombiforia L } \\
\text { PT047 }\end{array}$ & Orweza & $\mathrm{H}$ & $\begin{array}{l}\text { Grasslands } \\
\text { Farmland }\end{array}$ & L & -Misfortune & $\begin{array}{l}\text {-Macerated leaves } \\
\text { mixed with water } \\
\text { and the child } \\
\text { bathed }\end{array}$ & Not found \\
\hline Melastomataceae & $\begin{array}{l}\text { Dissotis } \\
\text { phaeotricha } \\
\text { (Hochst.) Hook. } \\
\text { f. PT024 }\end{array}$ & Mukurateitabye & $\mathrm{H}$ & Bush & $L$ & $\begin{array}{l}\text {-Malaria } \\
\text {-Fever } \\
\text {-Worms }\end{array}$ & -Decoction drunk & Not found \\
\hline Meliaceae & $\begin{array}{l}\text { Azadirachta } \\
\text { indica A.Juss. } \\
\text { PT007 }\end{array}$ & Nimu & $\mathrm{T}$ & Farmland & $\begin{array}{l}\mathrm{L} \\
\mathrm{Fr} \\
\mathrm{S}\end{array}$ & $\begin{array}{l}\text {-Worms } \\
\text {-Malaria } \\
\text {-Fever } \\
\text {-Skin rash } \\
\text {-Jaundice }\end{array}$ & $\begin{array}{l}\text {-Decoction and } \\
\text { infusion drunk } \\
\text {-Oil from seeds } \\
\text { drunk }\end{array}$ & $\begin{array}{l}\text { Decoction used for skin rash } \\
\text { in Ogun State Nigeria [28]; } \\
\text { cough, fever [26] }\end{array}$ \\
\hline Moraceae & $\begin{array}{l}\text { Ficus natalensis } \\
\text { Hochst. PT050 }\end{array}$ & Ekitooma & $\mathrm{T}$ & $\begin{array}{l}\text { Bush } \\
\text { Farmland }\end{array}$ & B & -Influenza & -Decoction drunk & Not found \\
\hline \multirow[t]{3}{*}{ Myrtaceae } & $\begin{array}{l}\text { Callistemon } \\
\text { citrinus (Curtis) } \\
\text { Skeels PT009 }\end{array}$ & $\begin{array}{l}\text { Bottle brush } \\
\text { (English) }\end{array}$ & $\mathrm{T}$ & $\begin{array}{l}\text { Home } \\
\text { compounds }\end{array}$ & $\begin{array}{l}\mathrm{L} \\
\mathrm{Br}\end{array}$ & $\begin{array}{l}\text {-Cough } \\
\text {-Common } \\
\text { cold }\end{array}$ & $\begin{array}{l}\text {-Decoction drunk } \\
\text {-Mixed with leaves } \\
\text { of Eucalyptus } \\
\text { grandis and } \\
\text { Mangifera indica } \\
\text { boiled and inhaled }\end{array}$ & Cough [19] \\
\hline & $\begin{array}{l}\text { Eucalyptus } \\
\text { grandis W. Hill } \\
\text { PT003 }\end{array}$ & Kalitusi & $\mathrm{T}$ & Farmland & $\begin{array}{l}L \\
B\end{array}$ & -Cough & $\begin{array}{l}\text {-Leaves chewed } \\
\text {-Infusion drunk } \\
\text {-Decoction drunk }\end{array}$ & Not found \\
\hline & $\begin{array}{l}\text { Psidium } \\
\text { guajava L. } \\
\text { PT015 }\end{array}$ & Omupera & $\mathrm{T}$ & Farmland & $\begin{array}{l}L \\
B\end{array}$ & -Cough & $\begin{array}{l}\text {-Decoction drunk } \\
\text {-Dried, pounded, } \\
\text { powder added to } \\
\text { cow ghee and } \\
\text { leaked }\end{array}$ & $\begin{array}{l}\text { Malaria, anaemia, fever, } \\
\text { diarrhoea [17] }\end{array}$ \\
\hline Passifloraceae & $\begin{array}{l}\text { Passiflora edulis } \\
\text { Sims PT027 }\end{array}$ & Obutunda & C & Farmland & $\begin{array}{l}R \\
T\end{array}$ & -Stomachache & -Decoction drunk & Diarrhoea, Cough $[17,19]$ \\
\hline \multirow[t]{2}{*}{ Poaceae } & $\begin{array}{l}\text { Cymbopogon } \\
\text { citratus Stapf } \\
\text { PT039 }\end{array}$ & Omutete & G & $\begin{array}{l}\text { Grassland } \\
\text { Farmland }\end{array}$ & $\begin{array}{l}L \\
S\end{array}$ & $\begin{array}{l}\text {-Diarrhoea } \\
\text {-Malaria } \\
\text {-Fever } \\
\text {-Ring worms }\end{array}$ & $\begin{array}{l}\text {-Decoction or } \\
\text { Infusion drunk } \\
\text {-Seeds crushed } \\
\text { mixed with water } \\
\text { and drunk }\end{array}$ & Yellow fever [19] \\
\hline & $\begin{array}{l}\text { Penissetum } \\
\text { perpureum } \\
\text { Schum. PT037 }\end{array}$ & Ekibingo & G & $\begin{array}{l}\text { Grasslands } \\
\text { Farmland }\end{array}$ & $\begin{array}{l}L \\
R\end{array}$ & $\begin{array}{l}\text {-Blindness } \\
\text {-Skin diseases }\end{array}$ & $\begin{array}{l}\text {-Infusion used to } \\
\text { wash the face } \\
\text {-Infusion drunk }\end{array}$ & Heart disease [19] \\
\hline \multirow[t]{2}{*}{ Rubiaceae } & $\begin{array}{l}\text { Pentas carnea } \\
\text { Benth. PT033 }\end{array}$ & Ruhaya & S & Bush & L & -Stomachache & -Decoction drunk & Not found \\
\hline & $\begin{array}{l}\text { Spermacoce } \\
\text { princea K. } \\
\text { Schum PT041 }\end{array}$ & Emarabyona & $\mathrm{H}$ & $\begin{array}{l}\text { Along foot } \\
\text { paths }\end{array}$ & Wp & $\begin{array}{l}\text {-Stomachache } \\
\text {-Worms }\end{array}$ & -Decoction drunk & Not found \\
\hline Rutaceae & $\begin{array}{l}\text { Citrus sinensis } \\
\text { (L.) Osbeck } \\
\text { PT022 }\end{array}$ & Omuchungwa & $\mathrm{T}$ & Farmland & L & $\begin{array}{l}\text {-Cough } \\
\text {-Sore throat }\end{array}$ & $\begin{array}{l}\text {-Leaves chewed } \\
\text {-Mixed with other } \\
\text { herbs, boiled and }\end{array}$ & Diarrhoea [17] \\
\hline
\end{tabular}


Table 1 Medicinal plant species, their habit, habitat, parts used, ailments treated, mode of preparation and administration (Continued)

\begin{tabular}{|c|c|c|c|c|c|c|c|c|}
\hline Family & $\begin{array}{l}\text { Scientific } \\
\text { name, } \\
\text { Voucher No. }\end{array}$ & $\begin{array}{l}\text { Local name } \\
\text { (Runyankole/ } \\
\text { Rukiga) }\end{array}$ & Habit & Habitat & $\begin{array}{l}\text { Parts } \\
\text { used }\end{array}$ & $\begin{array}{l}\text { Ailment } \\
\text { treated }\end{array}$ & $\begin{array}{l}\text { Method of } \\
\text { preparation \& } \\
\text { administration }\end{array}$ & $\begin{array}{l}\text { Literature supporting } \\
\text { traditional use of the } \\
\text { plant species in } \\
\text { other regions. }\end{array}$ \\
\hline & & & & & & & decoction drunk & \\
\hline \multirow[t]{4}{*}{ Solanaceae } & $\begin{array}{l}\text { Datura innoxia } \\
\text { Mill PT026 }\end{array}$ & Nyarweziringa & S & $\begin{array}{l}\text { Swamps } \\
\text { Farmland }\end{array}$ & $\begin{array}{l}L \\
S \\
F r \\
S\end{array}$ & $\begin{array}{l}\text {-Cough } \\
\text {-Asthma } \\
\text {-Skin diseases }\end{array}$ & $\begin{array}{l}\text {-Infusion is drunk } \\
\text {-Macerated leaves } \\
\text { are applied on skin }\end{array}$ & Not found \\
\hline & $\begin{array}{l}\text { Nicotiana } \\
\text { tobacuum L. } \\
\text { PT029 }\end{array}$ & Etaabe & $\mathrm{H}$ & Farmland & $L$ & $\begin{array}{l}\text {-Stomachache } \\
\text {-Common } \\
\text { cold } \\
\text {-Influenza }\end{array}$ & $\begin{array}{l}\text {-Leaves } \\
\text {-Leaves are } \\
\text { macerated and } \\
\text { juice given to the } \\
\text { child }\end{array}$ & Snake bites, Migraine [26] \\
\hline & $\begin{array}{l}\text { Physalis } \\
\text { peruviana L. } \\
\text { PT044 }\end{array}$ & Ekituutu & C & $\begin{array}{l}\text { Bush Along } \\
\text { hedges }\end{array}$ & $L$ & -Stomachache & -Decoction drunk & $\begin{array}{l}\text { Hypertension, malaria, } \\
\text { menstrual pains, nausea, } \\
\text { splenomegaly,stomachache } \\
\text { [21] Vomiting [26] }\end{array}$ \\
\hline & $\begin{array}{l}\text { Solanum } \\
\text { incanum L. } \\
\text { PT034 }\end{array}$ & Entengotengo & $S$ & $\begin{array}{l}\text { Grasslands } \\
\text { Farmland }\end{array}$ & $\begin{array}{l}\mathrm{Fr} \\
\mathrm{L} \\
\mathrm{R}\end{array}$ & $\begin{array}{l}\text {-Wounds } \\
\text {-Toothache } \\
\text {-Prolapsed } \\
\text { rectum }\end{array}$ & $\begin{array}{l}\text {-Fruits are baked } \\
\text { and used to } \\
\text { massage the } \\
\text { rectum } \\
\text {-Juice from } \\
\text { macerated leaves } \\
\text { applied }\end{array}$ & Yellow fever [17] \\
\hline Verbanaceae & $\begin{array}{l}\text { Lantana trifolia } \\
\text { L. PT038 }\end{array}$ & Omuhukye & $S$ & $\begin{array}{l}\text { Bush } \\
\text { Farmland }\end{array}$ & L & -Fresh wounds & $\begin{array}{l}\text {-Infusion applied } \\
\text { topically }\end{array}$ & $\begin{array}{l}\text { Cough, amoebiasis [26];Sore } \\
\text { throat, general malaise \& } \\
\text { gonorrhoea [29] }\end{array}$ \\
\hline
\end{tabular}

Key: Habit: H - Herb, S - Shrub, T - Tree, C - Climber, Parts used: L - Leaves, R- Roots, Fr - Fruits, S- Seeds, Wp - Whole plant, B - Bark,. Habitat means areas where respondents sourced the plant species; Farmland represents cultivated land, banana plantations

disease treated, plant part(s) and mode of preparation and administration are presented in Table 1 . Asteraceae was the most dominant family and contributed eight species followed by Lamiaceae (7), Fabaceae (5), Solanaceae (4) and Myrtaceae (3). The other families had one to two species each.

\section{Growth forms, habitat and plant parts used}

Herbs formed the highest proportion of medicinal plant species (36\%) closely followed by shrubs (32\%), trees (22\%), climbers (6\%) and grasses (4\%). The various plants parts used in herbal remedy preparation included fruits, leaves, roots, seeds, stems and the bark. In some cases whole plants were used. Leaves were the most frequently used part (58\%), followed by roots (16\%). The use of other plant parts was less common and in the range of $2-5 \%$. However, there were instances where more than one plant parts of the same plant were used. For instance the leaves and roots of Vernonia amygdalina were used to treat malaria and worm infestation; leaves and stem bark of Spathodea companulata were used against allergy, dysentery, diarrhoea and asthma; seeds, leaves and roots of Dracaena fragrans were used to treat stomachaches while leaves and roots of Mimosa pudica were used against sores and abscesses.
For herbs, the whole plant was uprooted, leaves were plucked off from shrubs and for trees debarking was the main method of harvesting. Other methods of harvesting included digging out the roots, cutting young branches and picking seeds. Participants revealed that in instances where the species were rare, uprooting whole plants and digging out roots were avoided. They also reported controlled harvesting of plant parts in the range of 0.5-5.0 $\mathrm{kg}$ once or twice a week depending on the availability of the plant, severity of the ailment and age of the child.

Some plant parts were extracted from more than one habitat (Table 2). Farmlands were the most common sources of medicinal plant species with frequency of $47 \%$ followed by bushes $26 \%$. Only two plant species Datura innoxia and Centella asiatica were extracted from swamps.

\section{Ailments treated using medicinal plant species}

A total of 29 diseases were managed using medicinal plant species. The diseases were grouped into 10 categories of which digestive disorders, malaria and respiratory tract infections featured predominantly (Table 3). Species that treated the highest number of health conditions were; Bidens pilosa, Mormodica foetida, Leonotis nepetifolia and Azadirachta indica (Table 1). Some 
diseases were treated using a single plant species and in other cases a mixture of plant parts from different species were used. Monotherapy preparations were dominant compared to herbal concoctions. For instance jaundice was treated either using the roots of Vernonia lasiopas or leaves of Erlangea tomentosa; asthma using the whole plant of Euphorbia hirta, anal rectal prolapse using leaves of Chenopodium murale, constipation using leaves of Vernonia amygdalina, allergy using leaves of Erlangea cordifolia, epilepsy using leaves of Sesbania sesban and the root tuber of Euphorbia tirucalli as an antidote.

Ailments that were managed using a combination of parts from different plants were; fever using a mixture of leaves of Vernonia amygdalina and V. lasiopas or leaves of $V$. amygdalina and roots of Iboza riparia. Cough was treated using a mixture of leaves of Eucalyptus grandis, Mangifera indica and Callistemon citrinus. A mixture of leaves of $V$. amygdalina and Erlangea cordifolia were used to treat headache. Dysentery was treated using leaves of Chenopodium murale and $V$. amygdalina, toothache using leaves of $V$. lasiopas and Bidens pilosa.

\section{Method of preparation and administration}

The major methods of preparation of plant extracts were decoction, infusion, maceration and chewing (Fig. 1). Water was the main solvent used in preparation of herbal therapies. Concoctions involved mixing water with an assortment of different plant parts. Decoctions were commonly used (80\%) and were prepared by boiling plant materials in a specific quantity of water for 15-20 min and the mixture allowed to cool before administration. Decoctions were followed by infusions (38\%) that involved pouring hot/warm water onto the plant material and allowing the mixture to cool. Maceration was used in $22 \%$ of the plant species. It involved crushing plant materials of a single species or a combination of more than one plant parts from different species

Table 2 Frequency of occurrence of medicinal plant species in different habitats

\begin{tabular}{lll}
\hline Habitat & Frequency & \% Frequency \\
\hline Farmland & 34 & 47.2 \\
Bush & 19 & 26.3 \\
Grasslands & 6 & 8.3 \\
Foot paths & 4 & 5.6 \\
Hedges & 3 & 4.2 \\
Home compounds & 3 & 4.2 \\
Abandoned lands & 2 & 2.8 \\
Swamps & 1 & 1.4 \\
Total & 72 & 100
\end{tabular}

Figures are inclusive of each other to extract a liquid which was applied either topically or orally. Minor methods of preparation like pounding and roasting were used with low frequencies in the range of $2-6 \%$ of the medicinal plant species.

Some remedies were prepared by pounding the plant material and applying the paste topically on the affected part. In other instances the paste was mixed with water and the resulting mixture drunk. For children below three years of age, the leaves were chewed by the mother of the child who spat the resulting mixture into the child's mouth. Children above three years of age chewed the leaves on their own. Some plant materials were roasted before applying them topically on the affected body parts. These were mainly used as poultices against abscesses, wounds and snake bites.

The modes of administration of herbal remedies included; leaking dry powdered plant materials especially leaves, drinking decoctions, infusions or concoctions, bathing the child with cold or warm extracts, applying crushed plant materials on the affected body parts, rubbing macerated plant materials on the affected part and mixing herbal preparations with food. Most respondents agreed that oral administration of herbal remedies was the most convenient for children.

There were similarities and variations in the use of plant species in the different villages in the study area. Some plant species were used in all the villages to treat the same ailments. For instance Mangifera indica and Psidium guajava for cough, Vernonia amygdalina against malaria, Dracaena fragrans and Ocimum sauve against stomach pains and Albizia adianthifolia against fever. In contrast, some plant species were used to treat different diseases in different villages. For instance Spathodea campanulata was used to manage asthma in Rutooma and Kishaka villages but used against dysentery in Mutanoga village. Aspilia africana was used to treat epilepsy in Oruhita village but used for diarrhoea in Kitookye and stomachache in Kishaka and Nyamuyaga villages.

\section{Discussion}

Asteraceae, Lamiaceae and Solanaceae which were dominant families in the current study are among the most reported families with species used in herbal remedy preparation in Uganda [21, 23, 30,31]. The high number of species from Asteraceae is attributed to the large number of its bioactive compounds [32]. Some of the plant species documented were used elsewhere in Uganda and other countries for treatment of either the same or different diseases (Table 1). The use of the same plant species to treat totally different conditions could be attributed to the fact that traditional knowledge is a closely guarded secret [33]. The use of same plant species for the same ailment in different localities indicates 
Table 3 Disease categories treated by different medicinal plant species

\begin{tabular}{llll}
\hline Disease category & Paediatric diseases & No. of plant species used $(N=50)$ & $\%$ of total species \\
\hline Digestive disorders & Stomachaches, diarrhoea, constipation, dysentery, & 33 & 66 \\
& worm infestation & 12 & 24 \\
Malaria & Malaria & 12 & 22 \\
Respiratory tract infections & Sore throat, influenza, cough, common cold, asthma & 11 & 6 \\
Other infections & Ear, eye \& oral infections & 3 & 18 \\
Skin infections & Wounds, ringworms, skin rash, allergy & 9 & 6 \\
Neurological disorders & Epilepsy, convulsions & 3 & 6 \\
Headaches & & 3 & 2 \\
Inflammation & Abscesses, boils & 1 & 2 \\
Poisoning & & 1 & 2 \\
Snake bites & & 1 & 2 \\
Misfortune & & 1 & \\
\hline
\end{tabular}

their cosmopolitan distribution and the fact that such plant species are effective for treatment of the specific ailments.

The high usage of herbs could be an indication of their abundance in the study area. These results are contrary to the findings of Kipkore et al. [34] and Katema et al. [35] where trees were dominant species for herbal medicine in Kenya and Ethiopia respectively.

The high frequency of occurrence of medicinal plant species in bushes is explained by the fact that plants that grow in the wild are rich in bioactive compounds [36]. Harvesting from farmlands and home gardens was prompted by the need to have medicinal plant species in the vicinity of homesteads to avoid travelling long distances in their search from the wild.

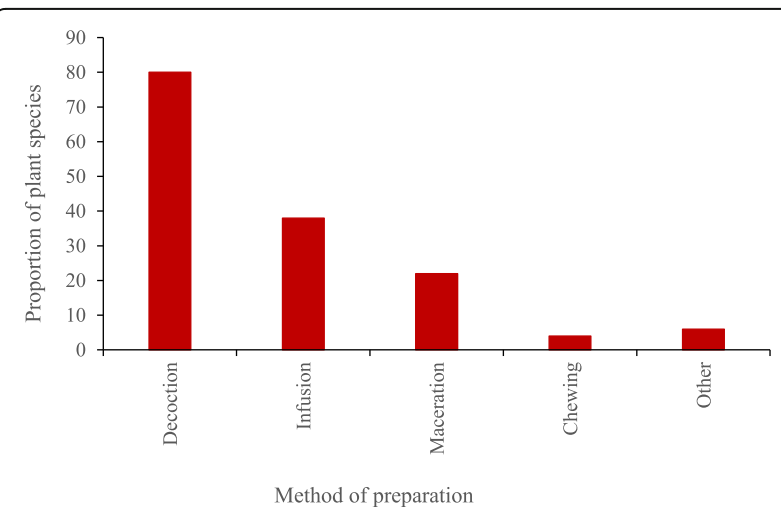

Fig. 1 The figure illustrates the percentage of medicinal plant species used for making herbal remedies using different methods according to information obtained from respondents. The total number of species for calculation of percentages was 50 . In some instances remedies from the same species could be prepared using more than one method. Water was the main ingredient used in preparation of herbal remedies in case decoctions and infusions. Method of preparation varied according to the plant species, parts used and sometimes conditions being treated
The use of leaves in herbal medicine preparation is common in other parts of Uganda [30,37], Thailand [38] and Bolivia [32]. Leaves are mostly used because of their potency and fast regeneration ability. They are the main photosynthetic organs and also act as storage for exudates or photosynthates; some of which are of medicinal value [39, 40]. Their dominant use could also be attributed to the ease with which they are harvested. The bark and young branches were the least used parts because preparation of remedies from these parts is hectic and the perception by local people that they contain fewer bioactive ingredients compared to leaves and roots.

The large number of plant species used to manage digestive system disorders is a clear manifestation that these conditions were the most predominant in children. This is attributed to poor sanitation which encourages prevalence of pathogens [41]. The prevalence of malaria in the parish can be attributed to bushes and stagnant water in the proximity of the homesteads that provided breeding grounds for mosquitoes [42]. The majority of the households did not use insecticide treated mosquito nets due to lack of money thus perpetuating the problem of malaria [43].

The use of a mixture of plants in herbal medicine preparation has been shown to increase the effectiveness of herbal remedies due to synergistic effects [23]. Decoctions were the most common method of herbal medicine preparation. Boiling aids extraction of active ingredients from medicinal plant parts and preserves the herbal remedy longer than using cold extraction [31]. However, in some cases boiling may cause severe degradation of bioactive ingredients especially the aromatic compounds if it takes a long time [44]. Some studies have reported preparation of herbal remedies as decoctions [45]. Though the use of herbal medicine was embraced in the study area, the problem of lack of 
standardisation of dosages was eminent as also reported in another study [46].

\section{Conclusions}

The people of Buhunga Parish have vast knowledge on medicinal plant species used in the treatment of various paediatric diseases. The medicinal plant species used are collected from a variety of habitats especially wild bush using several harvesting techniques some of which are destructive. The study creates awareness of the relevance of plant species in treatment of paediatric diseases, the therapeutic claims of which need to be assessed through phytochemical and pharmacological investigations to discover their bioactive ingredients. Further research focusing on preparation of herbal therapies with standardised dosages should be conducted in order to come up with recommended doses.

\section{Abbreviations}

IPNI: International Plant Names Index; WHO: World Health Organisation

\section{Acknowledgements}

We wish to thank the Local Council Chairpersons of the villages who authorised conducting the survey in their jurisdiction and the local people of Buhunga parish that provided information. We also acknowledge the assistance rendered by the staff of Makerere University Herbarium in identifying the plant species.

\section{Authors' contributions}

PT conceptualized the study, designed the methods, conducted the ethnobotanical survey and drafted the manuscript. CN conceptualized the idea of this manuscript and participated in reviewing the manuscript. Both authors read and approved the final manuscript.

\section{Funding}

Not applicable.

\section{Availability of data and materials}

All data generated and analysed during this study are included in the article.

\section{Ethics approval and consent to participate}

The work was approved by the Department of Plant Sciences, Microbiology \& Microbiology - Makerere University, Research Committee. Verbal prior informed consent was obtained from respondents before interview.

\section{Consent for publication}

Not applicable.

\section{Competing interests}

The authors declare that they have no competing interests.

Received: 15 February 2019 Accepted: 20 November 2019

Published online: 05 December 2019

\section{References}

1. Ghorbani A. Clinical and experimental studies on polyherbal formulations for diabetes: current status and future prospective. J Intergr Med. 2014;12: 336-45.

2. Sahoo N, Manchikanti P, Dey S. Herbal drugs: standards and regulation. Filoterapia. 2010;81:462-71

3. World Health Organisation Mental Health Global Action program (mHLGAP). 2002. http://who.int/medicines/publications/traditional/trm_strategy14_23/ en/index.html. Accessed 23 Nov 2018.

4. WHO.WHO strategy on Traditional Medicine 2014-2023. 2013. https://www. who.int/medicines/publications/traditional/trm_strategy14_23en. Accessed 7 Feb 2019.
5. Kamatenesi M. Utilisation of Medicinal plant "Nyakibazi" (Rytigynia sp) in multiple use zones of Bwindi Impenetrable National Park. (MSc.). Kampala: Makerere University; 1997.

6. Marshalls NT. Searching for a cure: conservation of medicinal wild resource in East and South Africa. In: Traffic Network Report. Cambridge: Traffic International; 1996.

7. Pfeiffer J, Butz R. Assessing cultural and ecological variation in ethnobiological research'; the importance of gender. J Ethnobiol Ethnomed. 2005;25(2):240-78

8. World Health Organisation. Maternal and child health: Uganda. Geneva: WHO; 2011

9. Republic of Uganda. Annual health sector performance. FInancial year 2014/ 2015. Kampala: Ministry of Health; 2015.

10. Ahmadipour SH, Mohsenzadeh A, Anban KH, Sadeghyar N. Iran's medical plants effective on fever in children/; a review. Annu Res Rev Biol. 2015;8(3):1-7.

11. Babaei A, Ansari MR, Alipour AA, Ahmadipour SH, Saferi-Faramani R, Vaikili ـ. A review of medicinal herbs that affects the kidney and bladder dtoned of children and adults in traditional medicine and ethnobotany of Iran. World Appl Sci J. 2012;18(5):600-4.

12. Colenbrander S, Birungi C, Mbonye AK. Consensus and contention in the priority setting process: examining the health sector in Uganda. Health Policy Plan. 2015;30 Suppl:555-65.

13. Kajungu D, Lukwago D, Tumushabe G. Assessing public expenditure governance in Uganda's Health sector: The case of Gulu, Kamuli and Luwero districts Policy Research Series. Kampala: ACODE; 2015.

14. Martin GJ. Ethnobotany: a methods manual. London: EarthScan; 2004.

15. Heinrich M, Edwards S, Moerman DE, Leonti M. Ethnopharmacological field studies: a critical assessment of their conceptual basis and methods. J Ethnopharmacol. 2009;124:1-17.

16. Katende AB, Birnie A, Tengnas B. Useful trees and shrubs of Uganda. Technical Handbook series 10. In: Regional Soil Conservation U/SIDA. Nairobi: Regional Land Management Unit RELMA/Sida ICRAF House, Gigiri; 1995.

17. Nalumansi P, Kamatenesi-Mugisha M, Anywar G. Medicinal plants used in Paediatric health Care in Namungalwe sub county, Iganga District, Uganda. Nov J Med Biol Sci. 2014;2(3):1-14.

18. Rabearivony AD, Kuhlman AR, Razafiarison ZL, Raharimalala F, Rakotoarivory F, Randrianarivory $\mathrm{T}$, et al. Ethnobotanical study of the medicinal plants known by men in Ambalabe, Madagascar. Ethnobot Res Appl. 2015;14:123-38.

19. Namukobe J, Kasenene JM, Kiremire BT, Byamukama R, Kamatenesi-Mugisha $M$, Krief S, et al. Traditional plants used for medicinal purposes by local comminities around the northern sector of Kibale National Park, Uganda. J Ethnopharmacol. 2011;136:236-45.

20. Oryem C, Ziraba RB, Omagor N, Opio A. Medicinal plants of Erute County, lira district Uganda with particular reference to their conservation. Afr J Ecol. 2010:48:285-98.

21. Asiimwe S, Namutebi A, Borg-Karlsson A, Kamatenesi-Mugisha M, OryemaOriga H. Documentation and consensus of indigenous knowledge on medicinal plants used by the local communities in WesternUganda. J Nat Prod PI Res. 2014;4(1):34-42.

22. De-la-Cruz H, Vilcapoma G, Zevallos PA. Ethnobotanical study of medicinal plants used by Andean people of Cata, Lima, Peru. J Ethnophamarcol. 2006; 111:284-94.

23. Tugume $\mathrm{P}$, Kakudidi EK, Buyinza M, Namaalwa J, Kamatenesi M, Mucunguzi $P$, Kalema J. Ethnobotanical survey of medicinal plant species used by communities around Mabira Central Forest Reserve, Uganda. J Ethnobiol Ethnomed. 2016;12:5.

24. Okello J, Ssegawa P. Plants used by communities of Ngai sub county, Apac District, Northern Uganda. Afr J Ecol. 2007;45(1):6-83.

25. Kshirsagar RD, Singh NP. Some less known ethnomedicinal uses from Mysore and Coorg districts, Karnataka State India. J Ethnopharmacol. 2001;75:231-8.

26. Tabuti JRS, Dhillion SS, Lye KA. Traditional medicine in Bulamogi conty, Uganda: its practitioners, users and viability. J Ethnopharmacol. 2003;85: $119-29$

27. Asiimwe S, Kamatenesi-Mugisha M, Namutebi A, Borg-Karlsson A, Musiimenta P. Ethnobotanical study of nutri-medicinal plants used for the management of HIV/AIDS opportunistic ailments among the local communities of Western Uganda. J Ethnophamarcol. 2013;150:639-48.

28. Shosan LO, Oluwasegu F, Ajiboye AA, Abeegunrin TA, Agboola DA. Ethnobotanical survey of medicinal plants used in curing some diseases in 
infants in Abeokuta south local government area if Ogun state Nigeria. Amer J PI Sci. 2014;5(21):3258-68.

29. Lulekal E, Kelbessa E, Bekele T, Yinegar H. An Ethnobotanical study of medicinal plants in Mana Angetu District, Southern Ethiopia. J Ethnobiol Ethnomed. 2008:4:10.

30. Bunalema L, Obakiro S, Tabuti JRS, Waako P. Knowledge on plants used traditionally in the treatment of tuberclosis in Uganda. J Ethnopharmacol. 2014;151:999-1004.

31. Kamatenesi MM, Acipa A, Oryema-Origa H. Medicinal plants of Otwal and Ngai sub counties in Oyam District, Northern Uganda. J Ethnobiol and Ethnomed. 2011;7:7.

32. Thomas E, Vandebroek K, Sanca S, Van Damme P. Cultural significance of medicinal plant families and species among Quechua farmers Apillapampa, Bolivia. J Ethnopharmacol. 2009;122:60-7.

33. Kigen GK, Ronoh HK, Kipkore WK. Current trends of traditional herbal medicine practice in Kenya: a review. Afr J Pharmacol Ther. 2013;2(1):32-7.

34. Kipkore W, Wanjohi B, Rono H, Kigen G. A study of the medicinal plants used by the Marakwet community in Kenya. J Ethnobiol Ethnomed. 2014;10:24.

35. Katema T, Etana D, Spiridoula A, Adugna T, Gebeyehu G, Jos GMH. Ethnomedical study of plants used for treatment of human and livestock ailments by traditional healers in south Omo, Southern Ethiopia. J Ethnobiol Ethnomed. 2013;9:32.

36. Guarrera PM, Savo V. Perceived health propertied of wild and cultivated food plants in local and popular traditions of Italy: A review. J Ethnopharmacol. 2013;146(3):659-80.

37. Ssegawa P, Kasenene JM. Medicinal plant diversity and uses in Sango bay area, Southern Uganda. J Ethnopharmacol. 2007;113:521-40.

38. Tangjitman K, Wongsawad C, Kamwong K, Sukicho T, Trisonthi C. Ethnomedicnal plants used for digestive system disorders by the Karen of northern Thailand. J Ethnobiol Ethnomed. 2015;11:27.

39. Ahmad M, Sultan S, Fazi-i-Hadi S, Hadda BT, Rashid S, Zafer M, Khan MA. An ethnoboatnical study of medicinal plants in high mountainous region of Chail Valley (district swat-Pakistan). J Ethnobiol Ethnomed. 2014;10:36.

40. Passalacqua N, Guarrera P, De Fine G. Contribution to the knowledge of the folk plant medicine in Calabria region (southern Italy). Fitoterapia. 2007;78:52-68

41. Bartram J, Cairncross S. Hygiene, sanitation and water: forgotten foundations of health. PLoS Med. 2010;7:e1000367.

42. Tabuti JRS. Herbal medicines used in the treatment of malaria in Budiope County, Uganda. J Ethnopharmacol. 2008;116:33-42.

43. Mugisha F, Arinaitwe J. Sleeping arrangements and mosquito net use among under-fives: results from Uganda demographic and health survey. Malaria. 2003:12:40

44. Moshi MJ, Otieno DF, Mbabazi PK, Weisheit A. Ethnomedicine of Kagera region, North Western Tanzania. Part 2: the medicinal plants used in Katoro Ward Bukoba district. J Ethnobiol Ethnomed. 2012;6:19-23.

45. Deeba F. Documentation of ethnoveterinary practices in urban and peri urban areas of Faisalabad Pakistan. (PhD). Faisalabad: University of Agriculture; 2009.

46. Alzweiri M, Sarhan AA, Mansi K, Hudails M, Aburjai T. Ethnopharmacological survey of medicinal herbs in Jordan, the northern Badia region. J Ethnobiol Ethnomed. 2011;137:27-35.

\section{Publisher's Note}

Springer Nature remains neutral with regard to jurisdictional claims in published maps and institutional affiliations.

Ready to submit your research? Choose BMC and benefit from:

- fast, convenient online submission

- thorough peer review by experienced researchers in your field

- rapid publication on acceptance

- support for research data, including large and complex data types

- gold Open Access which fosters wider collaboration and increased citations

- maximum visibility for your research: over $100 \mathrm{M}$ website views per year

At BMC, research is always in progress.

Learn more biomedcentral.com/submissions 